\title{
¿Podemos fiarnos de la evaluación en los sistemas de educación a distancia y digitales?
}

\section{(Can We Trust Evaluation in Distance and Digital Education Systems?)}

\author{
Lorenzo García Aretio
}

UNED (España)

DOI: https://doi.org/10.5944/ried.24.2.30223

\section{Cómo referenciar este artículo:}

García Aretio, L. (2021). ¿Podemos fiarnos de la evaluación en los sistemas de educación a distancia y digitales? RIED. Revista Iberoamericana de Educación a Distancia, 24(2), pp. 9-29. https://doi.org/10.5944/ried.24.2.30223

\section{Resumen}

Se prueba que la evaluación es una variable esencial en todo proceso de enseñanzaaprendizaje. Se enfatiza la necesidad de diseñar una evaluación integrada en el proceso formativo, sistemática y continuada, que, además de la preceptiva evaluación sumativa y final, nos permita, mediante una evaluación formativa, orientar el aprendizaje y juzgar alternativas previas a la toma de decisiones. Y estas destacadas características son válidas tanto en procesos educativos presenciales como en aquellos soportados en tecnologías digitales. Tanto en un caso como en el otro, se refuerza la relevancia y necesidad de la información de retorno al estudiante, feedback, de calidad, como un respaldo permanente a la reconducción hacia la mejora. Respecto a estas modalidades no presenciales, se justifican las variantes más habituales de tipologías de evaluación existentes, la autoevaluación y la coevaluación, que se muestran como complementos eficaces, tanto como estrategias de formación en sí mismas, como de estímulo para la mejora; las pruebas a distancia (síncronas o asíncronas), de las que se destacan en el trabajo numerosas ventajas y posibilidades; las pruebas presenciales, aconsejables en estudios formales y reglados, y otras variantes alternativas. Finalmente se apuntan una serie de ventajas que brindan las tecnologías para asegurar procesos evaluativos de calidad, tanto de carácter síncrono, necesarios en épocas de confinamiento, como asíncrono.

Palabras clave: evaluación a distancia; evaluación continua; evaluación formativa; evaluación síncrona y asíncrona. 


\begin{abstract}
It is proven that evaluation is an essential variable in any teaching-learning process. The need to design an integrated evaluation in the training process, systematic and continuous, is emphasized that, in addition to the mandatory summative and final evaluation, allows us, through a formative evaluation, to guide learning and judge alternatives prior to decision making. And these important characteristics are valid both in face-to-face educational processes and in those supported by digital technologies. In both cases, the relevance and need for quality feedback to the student is emphasized as a permanent support for redirection towards improvement. Regarding these non-face-to-face modalities, the most common variants of existing evaluation typologies are justified, self-evaluation and co-evaluation, that are shown as effective complements, both as training strategies in themselves, and as a stimulus for improvement; remote tests (synchronous or asynchronous), of which numerous advantages and possibilities are highlighted in this work; face-to-face tests, advisable in formal and regulated studies, and other alternative variants. Finally, a number of advantages that technology provide to ensure quality evaluation processes are pointed out, both of a synchronous nature, necessary in times of confinement, and of an asynchronous one.
\end{abstract}

Keywords: distance assessment; continuous assessment; formative assessment; synchronous and asynchronous assessment.

Todas las actividades humanas reciben de manera implícita o explícita una valoración, en unos casos para sancionar el resultado o rendimiento de una tarea y en otros, que parece son la mayoría, para realimentar el sistema al descubrir los fallos, lagunas o deficiencias del proceso. Siempre para tomar decisiones pertinentes que reconduzcan las acciones y refuercen la autorregulación (Allal, 1980) con el fin de mejorar el rendimiento o, en su caso, de aprobar o reprobar el resultado final o parcial (García Aretio, 1987).

El proceso de aprendizaje y, correlativamente, las estrategias de enseñanza conforman una actividad humana de primera magnitud cuyo protagonista es el sujeto que aprende y con otro sujeto no menos importante que existe, precisamente, para facilitar ese aprendizaje, el profesor. En esa actividad, obviamente, se hace igualmente precisa la medida, la valoración y toma de decisiones, es decir, la evaluación (Walvoord, 2010). Y debería evaluarse a través de criterios objetivos basados en parámetros que puedan indicarnos la consecución o no de las metas previstas (Shute y Becker, 2010) con el fin de orientar la consolidación de los puntos fuertes y el refuerzo en las lagunas o deficiencias observadas.

De ahí que esa evaluación, no debería ser una fase aislada y mucho menos, final, del proceso, sino integrada, formando parte de él como elemento sustancial del mismo (Pérez Juste, 1996; Brown, 2015). Y bien es sabido que una evaluación bien concebida puede implementarse para fomentar e impulsar el aprendizaje de los estudiantes (Pintrich y Zusho, 2002; Nicol, 2010). 
Por ello, desde hace años, puede entenderse la evaluación del aprendizaje como la acción de obtención de información sobre el estudiante y la naturaleza y calidad de su aprendizaje, integrada en el proceso formativo, sistemática y continuada, que nos permite orientar el aprendizaje y juzgar alternativas previas a la toma de decisiones (García Aretio, 1994, 2001). Y ahí se hace referencia a la evaluación de los aprendizajes, sean éstos obtenidos en formatos educativos presenciales o no presenciales. Si la evaluación siempre ha sido considerada como variable curricular fundamental, en las metodologías a distancia los procesos de evaluación de los aprendizajes se vuelven aún más relevantes y se configuran como instrumento determinante dentro de los procesos educativos (Schrum et al., 2007).

Lo que sucede, es que han venido siendo bastante habituales en los diferentes formatos de las modalidades educativas no presenciales, las prevenciones, dudas, temores, confusiones, malentendidos, etc., que se han generado en torno al concepto, objeto, contenido, fases, protagonistas, validez, fiabilidad, etc., de la evaluación de los aprendizajes, sobre todo cuando se trata de procesos de heteroevaluación.

Pues bien, por ahí irá el interés de este trabajo. Se tratarán de destacar 1los conceptos de evaluación continua ligada a la evaluación formativa, con énfasis en el feedback o realimentación, con el fin de reafirmar que esas características esenciales de la buena evaluación están presentes en los modelos a distancia de calidad.

\section{LA EVALUACIÓN CONTINUA, INTEGRADA Y FORMATIVA}

Una evaluación continua, según el momento o tiempo en que se realizase podría basarse en una diagnosis, evaluación inicial, apropiada para conocer las lagunas, posibilidades y limitaciones de cada estudiante o grupo, una evaluación procesual o continuada, realizada a lo largo del curso, integrada en el proceso, con recogida sistemática de informaciones parciales, y una evaluación final que debería ser consecuencia lógica de la procesual y más cercana a una evaluación global (García Aretio, 2001). Ha de superarse la mera idea de considerar a la evaluación como el examen, la calificación, la nota, la certificación, la promoción, la tarea educativa final, la simple medida y la decisión final, sin más. Y, lamentablemente, esto se continúa haciendo, es lo que prevalece en las aulas universitarias (Álvarez et al., 2011; San Martín et al., 2016), tanto en modalidades presenciales como a distancia.

Una evaluación continua, y por tanto integrada, con episodios y registros frecuentes en el tiempo, lleva a una retención y éxito más prolongados que las situaciones de evaluación a examen único (Kornell, 2009; Hussey, 2017). Si no se desarrollan en nuestra acción docente procesos de evaluación continua, resulta inútil hablar o escribir sobre logros de competencias, al menos en muchas asignaturas y cursos, a no ser que nos limitemos a valorar lo estrictamente memorizado por el estudiante. Los procesos de evaluación continua ayudan a los estudiantes a mantener el camino correcto y mejorar su rendimiento (Domenech et al., 2015; Day et al., 2017). 
Una evaluación continuada, a lo largo de todo el proceso, que haya previsto, diseñado, esas actividades que obligan y centran la acción en el que aprende, que le reconducen sobre sus previsibles errores (Coll et al., 2008), sí podría dar cuenta del logro o no de aquellas competencias/objetivos previstos y deseados también los de orden complejo y, además, permitiría a los docentes familiarizarse con el trabajo continuado de sus estudiantes (Russell et al., 2006), que les llevará a comprobar logros de objetivos complejos, obtenidos en varias etapas a lo largo del curso.

$\mathrm{Si}$ el fin de lo que se pretende es sólo averiguar el dominio conseguido por el estudiante con la finalidad de certificar o acreditar unos resultados o de asignar una calificación de aptitud o inaptitud referente a determinadas competencias, conocimientos, destrezas o capacidades adquiridos en función de las previsiones, se trata de medir lo aprendido, a base de acumular datos frecuentes e, incluso, sistemáticos, se estaría, sí, en una evaluación continua, pero que se denominaría sumativa. Podría arbitrarse un sistema de ponderaciones según los diferentes actos evaluativos y al final, generar un número, o una letra o palabra que calificase ese resultado.

Sin embargo si, además de una calificación final, el objetivo es proporcionar una información continuada, al estudiante (feedback), válida también para el docente, con el fin de, posteriormente, tomar decisiones; si se busca que el estudiante corrija defectos y confusiones, supere dificultades y adquiera competencias que se han detectado como ausentes en la prueba o trabajo de evaluación propuesto, se estaría más que midiendo lo aprendido, evaluando para aprender (Scriven, 1967; Morgan y O’Reilly, 1999; Earl, 2013), es decir, realizando una evaluación, además de continuada, formativa. Se trataría, como señala Barberà (2006), no sólo de evaluar los aprendizajes, sino evaluar para aprender tras el feedback, evaluar como aprendizaje en sí mismo o evaluar desde lo que ya se sabe.

Así las decisiones que hayan de adoptarse cuando se está seguro de haber llevado a cabo estos procesos formativos se mostrarán útiles, factibles, exactas, válidas, objetivas y éticas (Stufflebeanm et al., 2002; Quesada, 2006). Por tanto, recogida constante y sistemática de información sobre la situación y aprendizaje del sujeto con el fin de orientarle en el proceso, y ayudarle a superar las dificultades que se le pudieran presentar durante el estudio, o sea, evaluación continua (McAlpine, 2002) que, en este caso, sería además formativa. Y ello se trate de formatos educativos presenciales, a distancia, combinados o híbridos.

Y en cualesquiera de esos formatos, si la evaluación es importante, el feedback o información de retorno, no lo es menos (Rodríguez e Ibarra, 2011). Un estudiante puede soportar bien el estudio sin contacto presencial, pero le será muy difícil llevarlo a cabo sin la correspondiente realimentación o feedback (Gibbs y Simpson, 2009) de la evaluación formativa (Ryan et al., 2000). Por otra parte, nadie ha aprendido nada de una calificación, de una nota. Sin embargo, sí puede aprenderse de un breve informe sobre los errores cometidos (Hills, 1981), y esa información suele generar elementos positivos en los futuros aprendizajes y en la calidad de estos. 
La evaluación así se convierte en un excelente recurso para la realimentación, para la información de retorno o feedback, tras la realización de una determinada prueba, actividad o tarea (Ferguson, 2011; Tillema, 2009). De hecho, la evaluación continua, para ser a la vez formativa, se debería configurar como un feedback permanente entre docente y estudiante, que informe de las carencias y logros respecto a esa actividad realizada.

Sería esa la forma de propiciar una autorregulación en el estudio y redirigir al logro de las competencias y objetivos no alcanzados (Allal, 1980). Si no existiese el feedback, no habría evaluación formativa. Si no se muestra a los estudiantes los resultados de sus avances y no se les orienta hacia la mejora, la evaluación, educativamente hablando, serviría de poco (Black y Wiliam, 1998; Coll et al., 2008; Gikandi et al., 2011). El rendimiento académico y la retención o permanencia de los estudiantes queda favorecido por propuestas de evaluación formativa que enfatizan la realimentación (Vallés et al., 2011; Chetwynd y Dobbyn, 2011).

Por tanto, la información de retorno o feedback habría de pasarse al estudiante siempre que éste haya realizado un acto evaluativo, haya sido presencial (Espasa et al., 2018) o en línea (McCarthy, 2017). Y deberá cuidarse especialmente la forma de llevar a cabo este feedback (Bangert-Drowns et al., 1991) que debe procurarse que sea efectivo (Shute, 2008). Un feedback, que se debe pretender total, personalizado, motivador, inmediato y claro y, también en diferentes formatos, individual o grupal, síncrono o asíncrono, en texto, audio o imagen, etc. (García Aretio, 2020a).

En fin, la triada evaluación continua, formativa y realimentación eficaz conforman elementos ideales para elevar el rendimiento de los estudiantes (Nicol y Macfarlane-Dick, 2006).

\section{MODALIDADES HABITUALES DE EVALUACIÓN EN SISTEMAS A DISTANCIA}

Antes de acometer las modalidades más habituales de evaluación en los sistemas a distancia convendrá hacer alusión a la distinción de criterios a la hora de evaluar. Así puede hablarse de evaluación normativa y criterial (Glaser, 1963). La evaluación se puede limitar a criterios de grupo, con lo que la calificación del individuo queda en una posición relativa con respecto a los otros miembros del grupo, evaluación normativa. O puede referirse la evaluación a criterios conductuales, especificados previamente, de logro de competencias, de objetivos y/o contenidos, evaluación criterial (Popham, 1983). Y estas dicotomías valen tanto para formatos de evaluación presenciales, virtuales, síncronos o asíncronos.

Respecto a todo lo aportado hasta ahora, no se conocen estudios rigurosos que afirmen que llevar a cabo una evaluación integrada, continua y formativa, nunca puesta en duda en los modelos presenciales, no pueda tener, al menos, la misma eficacia y posibilidades en los entornos virtuales de enseñanza y aprendizaje. ¿Cuáles son las modalidades más habituales de evaluación en los estudios no presenciales? 


\section{La autoevaluación y la coevaluación}

Además de la tradicional y más habitual evaluación de los aprendizajes de los estudiantes por parte de sus profesores, heteroevaluación, existen otros tipos de evaluaciones alternativas, según el agente evaluador. Autoevaluarnos y valorar el trabajo de otros resulta una tarea casi permanente en la sociedad actual. Es una forma de involucrar a los propios estudiantes en la posterior toma de decisiones sobre su evaluación. Parece que implicándoles en estas formas alternativas de evaluar, negociando, evaluando a otros, etc., los estudiantes aprenden más y mejor y pueden elevar su autoestima (Falshikov, 1986).

En concreto, nadie mejor que el estudiante puede valorar el esfuerzo realizado, el tiempo dedicado, las dificultades superadas, la satisfacción o insatisfacción, etc., producidos por los aprendizajes. Se trataría de la autoevaluación. Ya se apuntó que los estudiantes son dados a identificar como importante para el aprendizaje y clave del estudio todo lo que se pregunta y cómo se pregunta, relegando a segundo término aquello que no fue motivo de autoevaluación. De esta manera se potenciará el repaso de lo fundamental de la unidad o tema, la puesta en práctica de determinadas habilidades y, también, se pueden detectar las partes más débiles del propio aprendizaje (McDonald, 2012).

Los ejercicios de autoevaluación o autocomprobación deberían ser un componente insustituible y muy cuidado en la educación a distancia, y ahora digital, por el valor de su realimentación inmediata (Robles y Braathen, 2002), acompañado de la correspondiente autorregulación, dado que, si están bien diseñados, el estudiante puede reaccionar al cerciorarse de la calidad de su progreso (Morgan y O’Reilly, 1999). Aprender a autoevaluarse, emitir juicios sobre el propio aprendizaje, supone aprender a ser más autónomo, es decir, adquirir un hábito de suma utilidad en diferentes facetas del futuro desempeño humano y un elemento importante para el objetivo del autoaprendizaje futuro (Thomas et al., 2011) y del permanente aprender a aprender.

Igual puede suceder cuando el proceso que se acomete es el de coevaluación, evaluación compartida (Deeley, 2014) o retroalimentación por pares (Panadero y Alqassab, 2019). En este caso, realimentan, enseñando sobre aciertos y errores, los propios pares que se convierten en juez y parte (Álvarez, 2008; Gessa, 2011). Uno mismo informa a los propios iguales, en función de la estrategia docente que se haya implementado, para llevar a cabo esa coevaluación. Resulta útil y conveniente que los propios pares que conforman el grupo, más allá de realizar su propia valoración, traten de observar el desempeño académico de los compañeros de curso y, por ello, también participen en el proceso como evaluadores y compartan la experiencia y comprueben sus propias limitaciones, a través de la coevaluación (Adachi et al., 2018; Dochy et al., 1999; Morgan y O’Reilly, 1999; Chien et al., 2020). Así, de la autorregulación puede pasarse a la corregulación. 
En este punto debería destacarse que no se puede afirmar que los sesgos en las puntuaciones, en estudios superiores, sean significativos, cuando se comparan propuestas de autoevaluación (Keveresky, 2017; Salvadores-Merino, 2019), evaluación entre iguales y heteroevaluación (Sánchez et al., 2017; Oren, 2018). Por otra parte, la evaluación por pares ofrece una retroalimentación eficiente y efectiva para los estudiantes (Topping, 2009), y tanto la autoevaluación como la coevaluación, llegan a empoderar a los estudiantes (Nulty, 2011).

\section{Las evaluaciones o pruebas a distancia}

En los sistemas a distancia fueron siempre habituales las pruebas de evaluación a distancia que se realizan con espacio y situación libre para el estudiante, con fecha límite o no para entregar o cumplimentar el trabajo o prueba y ahora, con los apoyos digitales, en tiempo síncrono, en directo, asíncrono y en línea. Estas pruebas pueden ser estructuradas de muy diversas maneras, siempre en función de la naturaleza del curso que se imparte y de las competencias y objetivos previstos. Desde trabajos amplios que requieran poner en juego diversas capacidades cognitivas del estudiante, no meramente memorísticas, pruebas de ensayo o actividades o trabajos de elaboración, sean estos teóricos o prácticos, que evalúan distintos objetivos de formación y logro de competencias, hasta pruebas objetivas plenamente automatizadas.

Es aconsejable utilizar instrumentos de evaluación múltiples -incluso dentro de cada unidad de evaluación- que pueden ser válidos sin que creen especiales problemas para ser resueltos en línea. Nos parece que esta forma de evaluación del proceso o evaluación continua facilita enormemente la evaluación formativa (Allal, 1980) a través de actividades, trabajos y pruebas que garantizan un estudio sistemático y eficaz y comportan otra serie de ventajas (Blair y Valdez, 2014) que se sugieren (García Aretio, 1987 y 1994), ahora modulados por las tecnologías:

- Suponen un control periódico e identificación del progreso académico de los estudiantes, posibilitando una evaluación continua a la vez que formativa.

- Obligan a estudiar, dado que puede ser preceptiva la cumplimentación de la prueba en una fecha determinada.

- Propician una adecuada distribución y organización del tiempo de trabajo, siempre que estén bien planificadas cronológicamente, en función del diseño del curso.

- Ayudan a la planificación del trabajo, dado que este estudio lo realizará el alumno de forma sistemática, ya que las pruebas se ajustan a la parte de la materia que se calcula debe haberse aprendido en una determinada unidad de tiempo, evitando así la sobrecarga de estudio en unas fechas determinadas.

- Ayudan a retener, comprender, aplicar, analizar, valorar..., los aspectos fundamentales de la materia. El estudiante habrá de insistir en los puntos no 
suficientemente asimilados que son motivo de actividad o pregunta, con el fin de alcanzar las competencias y objetivos que se pretenden, y de esta manera, convertirse en auténtico protagonista de su aprendizaje.

- Se utilizan como elemento de comunicación bidireccional, información de retorno o feedback (evaluación formativa) dado que habrán de ser valorados por los correspondientes docentes con la corrección de errores, calificación y las pertinentes orientaciones que sugieran estrategias de estudio enfocadas al logro de los saberes o destrezas no alcanzados.

- Promueven la participación y el aprendizaje activo, dado que el conocimiento, por parte del estudiante, de los resultados de su aprendizaje y la correspondiente orientación sobre el mismo, actúa como guía y fuerte incentivo para el pensamiento crítico y la autorregulación, mejora en organización, cantidad y calidad de los futuros logros académicos.

- Se podrían utilizar también como instrumento para la coevaluación, o evaluación entre pares. En estos casos se hace más necesaria la elaboración de rúbricas bien ajustadas a los procesos que pretenden medirse.

- Se fuerza al alumno a no limitarse en su estudio a la consulta del material estrictamente obligatorio, al solicitársele respuesta a cuestiones que requieran la reelaboración, análisis de enfoques distintos o relación entre ellos, etc., que ponen al sujeto en la necesidad de consultar otras fuentes bibliográficas o a profundizar en las recomendadas como básicas.

- Se puede exigir, en algunos casos, la postura personal en determinadas cuestiones, que requerirán la reflexión y el estudio con detalle de todo tipo de material.

- Sirven de entrenamiento -en su caso- para la realización de las pruebas presenciales, dado que algunas de ellas deberían estar estructuradas en formato similar, en cuanto al contenido y estilo de las cuestiones, aunque requieran un tiempo superior para su cumplimentación que las presenciales.

- Orientan a los docentes para detectar los bloques de la materia en donde existen mayores lagunas en el aprendizaje y los fallos más notables, para poder insistir y reforzar los contenidos y procesos que se hagan necesarios. Y ello puede hacerse mediante orientaciones individuales o a través de refuerzos puntuales con materiales textuales o audiovisuales y, naturalmente, a través de los foros.

- Se establece el oportuno requisito académico de la cumplimentación de estas pruebas sin las cuales, en determinados programas, no puede superarse la materia o curso en cuestión.

A veces se configuran como el elemento exclusivo para la toma de decisiones, sin necesidad de implementar una evaluación final de carácter presencial. Por tanto, evaluación integrada en el proceso, continua y maximizando la realimentación (Meyen et al., 2002). 


\section{Las pruebas presenciales}

Una evaluación presencial consiste en la realización de pruebas o trabajos que habrán de desarrollarse con tiempo síncrono, espacio físico idéntico y situación delimitados con suficiente antelación (Shuey, 2002).

Son pruebas que, cuando se establecen, ponen al estudiante en disposición de demostrar hasta qué punto los ejercicios de autoevaluación y, sobre todo, las pruebas realizadas a distancia han sido fruto de su exclusivo esfuerzo personal. Este tipo de pruebas es recomendable, quizás todavía imprescindible, en aquellas materias y cursos cuya superación llevan al logro de una certificación, título o acreditación de carácter oficial. Hasta tanto las modernas tecnologías no garanticen formas de examen en las que sea muy difícil la suplantación de identidades, la deshonestidad, etc. (Rovai, 2000) y superados los posibles problemas técnicos, jurídicos y éticos (Aznarte y Lacruz, 2021), seguiría recomendando este tipo de pruebas en los casos señalados, además de una evaluación continua y formativa a distancia (evaluación mixta o híbrida). En todo caso, en situaciones de pandemia hay que arbitrar soluciones digitales que eviten al máximo conductas fraudulentas.

\section{Otras evidencias de evaluación en los sistemas a distancia}

No deberían considerarse como «pruebas» propiamente dichas, pero sí como elementos o evidencias que nos permitirán acumular información con respecto al sujeto de evaluación. De ahí, la participación en las diferentes actividades interactivas síncronas y asíncronas que posibilitan las plataformas (foros, chat, wikis...), la calidad y cantidad de estas participaciones, los documentos leídos, escuchados o visionados... La analítica de aprendizajes, puede ayudarnos a enfocar hacia un aprendizaje adaptativo, mediante la mejora de diseños y de una más eficaz realimentación (Martin y Ndoye, 2016).

Hoy día muchas plataformas digitales facilitan esas posibilidades de autoevaluación, coevaluación, heteroevaluación (Anderson, 2008), evaluación síncrona y asíncrona, además de poder llevar a cabo estudio de los datos o analítica de aprendizajes. Nunca en los formatos presenciales se podrá contar con tal cantidad de registros y evidencias relativos al aprendizaje de los estudiantes.

\section{SOBRE LAS DUDAS Y CAUTELAS RESPECTO A LA EVALUACIÓN EN LOS SISTEMAS VIRTUALES}

Con la autoridad que nos ofrecen los autores consultados y referenciados más arriba, y basado en trabajos anteriores, modulados por las tecnologías digitales (García Aretio, 1994, 2001), podría afirmarse que el tiempo dedicado a una buena planificación de la evaluación comportará una serie de ventajas respecto a reforzar las 
funciones básicas de la misma en todo el proceso de diseño y desarrollo educativos, válidas tanto para procesos presenciales como para aquellos de corte digital. Éstas serían, a modo de síntesis, esas funciones.

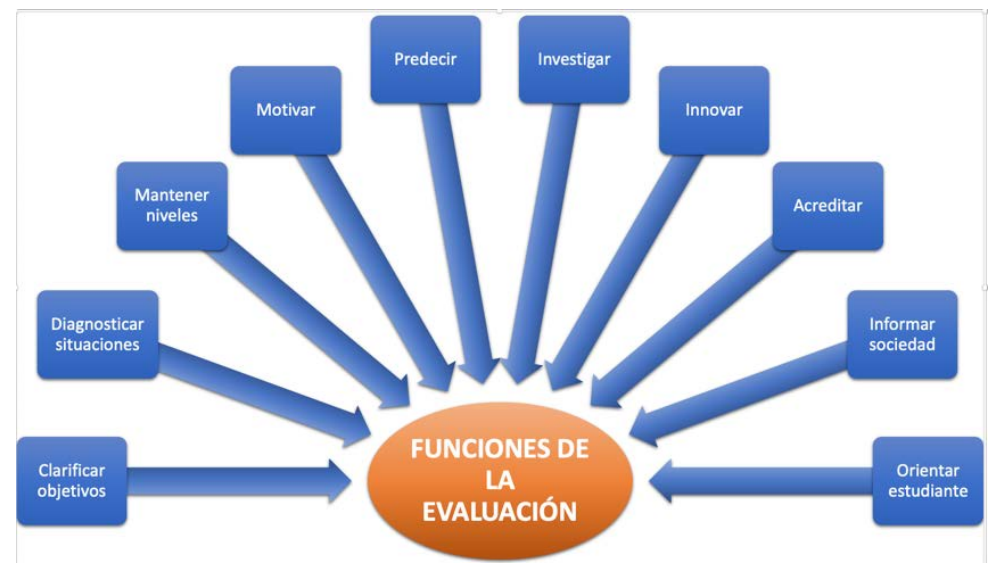

- Clarificar y ajustar objetivos y contenidos relacionados con las competencias. ¿Qué evaluar?, ¿para qué?, ¿son competencias, son objetivos y contenidos apropiados, claros, realistas, evaluables...?

- Diagnosticar situaciones. Bien sean de individuos concretos o del grupo de estudiantes que siguen el curso, con el fin de reconducir, si es preciso, el proceso de enseñanza a sus posibilidades y limitaciones.

- Mantener constantes los niveles académicos. La evaluación sirve de guía al propio docente para cerciorarse sobre el rendimiento de los estudiantes y sobre las previsibles desviaciones del itinerario formativo previsto que pudieran llevar a metas no deseadas.

- Motivar pautas de actuación de estudiantes y profesores. Una evaluación bien concebida y diseñada indica al estudiante qué, cómo y cuándo se estudia, y al profesor qué y cómo se enseña, con el fin de facilitar el éxito y permanencia y huir del fracaso y abandono.

- Predecir resultados. La prevención y el pronóstico facilitados por una evaluación inicial y del proceso son más pedagógicos que la recuperación posterior de lo no aprendido al facilitar información que nos permite actuar con inmediatez.

- Propiciar la investigación. Con los datos recogidos pueden compararse métodos y estrategias, cursos, grupos de alumnos, diseños, recursos, etc.

- Fundamentar la innovación. La investigación referida puede probar la eficacia de nuevos métodos o estrategias didácticas que inviten a la introducción de algún cambio que transforme y mejore la estructura o elementos de la acción formativa. 
- Acreditar socialmente unos resultados. Enfocados a la toma de decisiones relativa a la promoción o no, a la obtención de un determinado reconocimiento académico o no, etc.

- Proporcionarinformaciónmásalládeldocente. Bien seaparala propiainstitución que imparte el curso, bien para instancias superiores (administraciones públicas, asociaciones, empresas, etc.), con el fin de tomar decisiones dirigidas a la mejora de la acción.

- Informar y orientar al estudiante (feedback), con el propósito de hacerle partícipe de sus deficiencias o progresos y así ayudar a fijar mejor sus aprendizajes futuros.

De poco servirá plantearse hermosos objetivos e inmejorables contenidos si se prescinde de su evaluación. El resultado de un buen curso puede quedar malogrado por un inadecuado enfoque de la evaluación o de sus técnicas. Los estudiantes no pueden escapar a esa realidad y si se les evalúa mal, aprenderán mal, aunque se les haya enseñado bien. En consecuencia, un curso o una asignatura sean presenciales o a distancia, pueden quedar muy determinados por lo que se evalúa.

Señaladas ya las posibilidades de una evaluación bien planificada para el logro de aprendizajes valiosos, en cualquier modalidad educativa, quizás haya de profundizarse más en las características propias de la evaluación en sistemas digitales. Así, para los más escépticos, habrían de mostrarse una serie de ventajas o posibilidades de las modalidades a distancia mediadas por tecnologías, que generalmente, facilitan contextos de evaluación enriquecidos. Estas son (García Aretio, 2017; Burns, 2018):

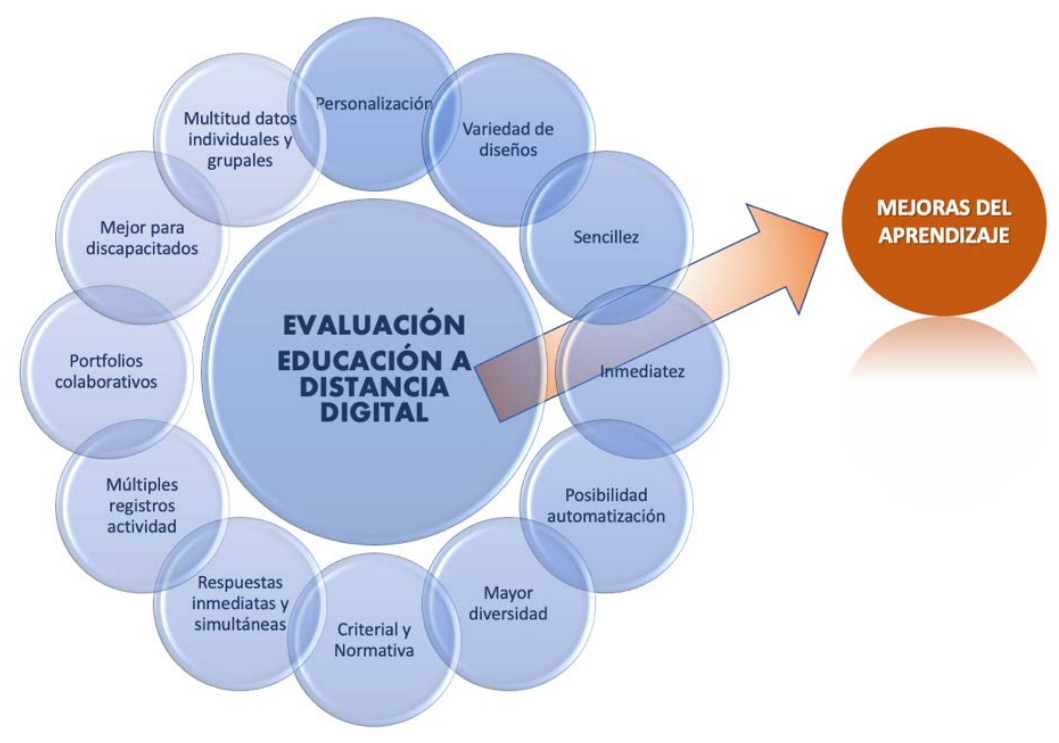


- Una mayor personalización del proceso evaluativo, evaluación adaptativa, preguntas secuenciadas por dificultad, etc.

- Gran variedad de diseños y presentaciones. Así en lugar de una sola prueba, se pueden realizar varias, secuenciadas en tiempos diferentes, síncronas o asíncronas.

- Resulta sencillo realizar una evaluación diagnóstica previa mediante pruebas en línea.

- En pruebas objetivas la calificación puede ser automatizada e inmediata y en las de respuesta abierta, cabe la posibilidad de automatizar una serie de rúbricas (García Aretio, 2020b). Pueden evitarse así los errores humanos.

- Mayor diversidad de técnicas e instrumentos de evaluación formativa continuada gracias al feedback automático.

- Se facilitan enfoques tanto para una evaluación criterial (superación de competencias u objetivos) como normativa (posibilidad de comparación entre los logros de los estudiantes).

- Se pueden obtener respuestas de todos los estudiantes a cualquier pregunta que pueda plantearse en los materiales de estudio, con el fin de conocer el progreso global del grupo.

- Múltiples registros de evidencias, de actividad del estudiante en la plataforma: inicios de sesión, tiempos de permanencia, lecturas de documentos, participaciones en foros, etc., que proporcionan amplia información para la toma de decisiones.

- Posibilidad de realizar portfolios en línea y seguimiento de la realización de actividades, así como el uso de herramientas tecnológicas para el trabajo colaborativo, la realización de prácticas en entornos simulados o remotos controlados por tecnologías, etc.

- Se podría asegurar una mejor atención a estudiantes con algún tipo de discapacidad visual, auditiva o motriz: lectores de pantalla, herramientas de ampliación, conversores de texto a voz o de voz a texto.

- La facilidad de realizar numerosas pruebas permite el acceso a un número sustancialmente mayor de datos individuales y grupales (analítica de aprendizajes).

A pesar de todo esto, en las modalidades no presenciales, se ha dudado mucho respecto a uno de los parámetros, al del control de la evaluación y a las posibilidades de fraude (García-Peñalvo, 2020; Harper et al., 2021). Aunque, respecto a las garantías en cuanto a la identidad de los estudiantes y la autoría de las actividades entregadas, conviene resaltar que en todas aquellas actividades que no se realizan delante de un profesor y en un entorno controlado, el fraude en los exámenes, el plagio o la suplantación de identidad en determinados trabajos entregados, son problemas comunes y similares, sea en entornos presenciales o a distancia. 
Es decir, en la mayor parte de actividades de evaluación fuera de los exámenes presenciales, las dificultades y, por tanto, los niveles de incertidumbre son los mismos en ambas modalidades (Dawson, 2016). Más allá de las posibilidades de la videoconferencia, los videomensajes, la foto, la identificación facial o de huellas..., que pueden dar fe de esa identidad (García Aretio, 2017).

Y, además, no existen más conductas deshonestas en las pruebas en línea que en las pruebas presenciales (Harmon y Lambrinos, 2008), siempre que estén diseñadas adecuadamente. El introducir evaluaciones electrónicas, sean de autoevaluación, coevaluación o heteroevaluación, lleva a aumentar considerablemente la actividad de los estudiantes en el entorno virtual de aprendizaje y su rendimiento (Holmes, 2017; McLaughlin y Yan, 2017).

Lo que sucede es que al profesorado le ha inquietado mucho más toda la problemática de la identidad del "examinado", que arbitrar medidas pedagógicas para que los estudiantes alcancen las competencias programadas (Sangrá, 2020). Muchos docentes no quieren, de ninguna manera, perder el control del proceso que, entienden, está en la evaluación (García Peñalvo, 2020). Estas exigencias confrontan a veces con todo lo relacionado con el derecho a la privacidad y las normativas sobre protección de datos (Aznarte y Lacruz, 2021). También se han arbitrado posibilidades de utilizar un alias que permitiese la anonimización de los estudiantes (Amo et al., 2020).

\section{UNA EVALUACIÓN SÍNCRONA Y EN LÍNEA}

¿Y qué sucedió con el confinamiento respecto a esta cuestión? Hubo que cambiar metodologías, recursos, sistemas de comunicación e interacción, etc. Y se hizo, mejor o peor. La enseñanza virtual en esos meses no era una opción, fue una necesidad e, incluso, una obligación (Dhawan, 2020). Sin embargo, parece que cambiar la evaluación supone mayores resistencias por parte de los docentes que introducir cambios en otras variables (Blázquez et al., 2017). Así, la evaluación online ha venido siendo un serio problema para muchos críticos (Friedman et al., 2016), sobre todo, como ya se indicó, en lo referido a la identificación de la persona evaluada por el riesgo de fraude o deshonestidad (Prince et al., 2009). Unas evaluaciones diseñadas inicialmente para ser propuestas con libro cerrado y sin recursos didácticos disponibles, se iban a hacer sin el control presencial. Esa era, en definitiva, la preocupación principal y el reto.

Lo más sencillo habría sido considerar que se ha venido realizando a lo largo del curso una rigurosa evaluación continua, tanto formativa como sumativa (Sambell et al., 2018), y que ello se entendiese como suficiente, o al menos determinante, para la toma final de decisiones.

Se ha avanzado enormemente en la propuesta de herramientas de vigilancia automática de exámenes, de control biométrico (proctoring), de reconocimiento facial, también dactilar, como garantía de identificación del estudiante (Adkins 
et al., 2005). No parecería excesivamente complicado integrar estos sistemas de identificación dentro de algunas plataformas virtuales modernas. Estos sistemas proctoring, o e-proctoring, podrían detectar con cierta fiabilidad si existiese algún tipo de suplantación de la persona que realiza el examen, presencia de elementos distintos a los necesarios para el examen, de otras personas, alteraciones de audio, visión $360^{\circ}$, el bloqueo del navegador, interoperabilidad con la plataforma virtual, diversas compatibilidades, etc. (González et al., 2020; García-Peñalvo et al., 2020).

Sin embargo, Aznarte y Lacruz (2021) apuntan a que existen evidencias de que estas tecnologías conllevan problemas técnicos, jurídicos y éticos. No en todos los países existe un marco regulatorio claro para el uso de tecnologías biométricas. Ha de combinarse la necesidad y la proporcionalidad, en el sentido de encontrar medidas menos gravosas que puedan aportarnos un resultado equivalente. Podría violarse el derecho a la privacidad. Más allá de que estas tecnologías de vigilancia automática son, a veces y todavía, imprecisas y el software falible; podrían producir discriminaciones y vulneraciones del principio de igualdad y podrían generar discriminación por motivos de diversidad funcional. Estos autores (Aznarte y Lacruz, 2021) afirman, además, que ni siquiera estas tecnologías pueden garantizar la autoría ni la ausencia de uso de medios fraudulentos en la realización del examen online.

Así, con las tecnologías con que se puede contar hoy, y prescindiendo del proctoring, en su momento se esbozó una serie de recomendaciones o variables a tener en cuenta para propiciar una evaluación más válida y fiable (García Aretio, 2020c). Algunas de estas sugerencias de tecnologías o de estrategias docentes, podrían tener alguno de los problemas señalados para las tecnologías de proctoring. En todo caso, estas sugerencias pueden plantearse como de aplicación simultánea, es decir, no tienen por qué ser excluyentes unas de otras:

- Videoconferencia, con identificación mediante el documento oficial de identidad existente en cada país, con herramientas tipo: Teams, Zoom, Skype, Blackboard, Google Meet, AVIP, etc. Pruebas orales o escritas. En este último caso (escritas), sin necesidad del reconocimiento facial, pero con plano fijo, garantizando mientras dura el examen, que la persona que opera en ese ordenador es el estudiante motivo de la prueba.

- Análisis síncrono, con capturas de vídeo, de las condiciones de la prueba.

- Fotografía. Toma aleatoria en tiempos diferentes de fotografía del estudiante que realiza la prueba. Debería facilitarse al inicio y/o final de la prueba, igualmente, una foto del documento de identidad.

- Control aleatorio remoto del ordenador desde el que se realiza la prueba.

- Bloqueo del ordenador para impedir el acceso a ayudas o a otras aplicaciones diferentes a la de la realización del examen.

- Simple control de acceso a la plataforma con el ID de usuario y la correspondiente contraseña. 
- Para limitar o reducir el riesgo externo de ayuda:

- Dar mayor alcance y peso a la evaluación continua con el fin de reducir los efectos de un examen final que pudiera ser fraudulento.

- Plantear preguntas que se alejen de la mera memorización o recuerdo.

- Un número suficientemente amplio de preguntas, especialmente en pruebas objetivas, que dificulta perder demasiado tiempo.

- Posibilidad de aleatorizar estas preguntas e, incluso, las alternativas de respuesta, en su caso. Ello exige contar con bancos o bases de preguntas muy amplias para no repetirlas en posteriores exámenes.

- Presentar en la aplicación de examen las preguntas aleatorizadas una a una, o por bloques, no todas a la vez, indicando el número de intentos de respuesta permitidos.

- Tiempo concedido y bien ajustado para responder que, infelizmente, puede perjudicar a los más lentos a la hora de teclear en las pruebas de desarrollo.

- Proponer cuestiones complejas que permitan utilizar material (libro abierto) $\mathrm{y}$ otros recursos. Estas preguntas han de estar muy bien planteadas y deben exigir poner en juego habilidades cognitivas de orden superior.

- Impedir la posibilidad de realizar copia/pega desde otros entornos a la pantalla de respuestas.

- Prueba oral. En la que se verifique previamente la identidad del estudiante y se pueda constatar que, al menos, el ordenador que incorpora la webcam no está ofreciendo material fraudulento (compartir escritorio, por ejemplo), y que la webcam ofrece una perspectiva amplia de la estancia y no sólo el rostro del examinado.

- Preguntas abiertas que también podrían responderse síncronamente a través de chat, foro u otra aplicación. Ligado con la siguiente.

- Antiplagio. Trabajos o realización de pruebas síncronas o asíncronas en los que se permite la utilización de todo tipo de material (pruebas de libro abierto) y que posteriormente se verán sometidos a la correspondiente herramienta del antiplagio.

- En estos últimos casos, y dependiendo del número de matriculados, también podría someterse al estudiante a una breve y posterior prueba oral que pudiera acreditar sus aprendizajes relativos a la tarea realizada.

De todas formas, habría de trabajarse por cambiar el enfoque de una evaluación que hasta ahora primaba el memorismo, y afianzar otros procesos educativos enfocados a poner en juego tipos de habilidades, tales como la comprensión, el análisis, la síntesis, la aplicación, la creación, etc., de esta forma el estudiante se habrá habituado a memorizar menos y a desarrollar otras capacidades que invitarían a ser más honestos y huir del plagio o fraude. Sería también una manera de ir 
conformando en los estudiantes, conductas más decentes. Por ejemplo, en estas circunstancias, exámenes de libro abierto serían plenamente factibles.

Por tanto, más que dedicar tanto tiempo y recursos a encontrar e implementar nuevas tecnologías de identificación y control, podríamos dedicar alguna parte importante de ese tiempo a diseñar nuevos enfoques de una evaluación auténtica, variada, continua, formativa, con pruebas y registros más frecuentes, etc., que así aleje las tentaciones de fraude.

\section{CONCLUSIÓN}

Queda probado que la evaluación es una variable crítica en los procesos de enseñanza-aprendizaje que, bien diseñada y aplicando las modalidades y estrategias apropiadas en cada caso, se convierte en un instrumento ideal para el logro de las competencias y objetivos propuestos. Ello, más allá de la función de medida, control o certificación que pudiera ofrecer según las circunstancias.

Todas las ventajas de la evaluación en los formatos educativos convencionales y presenciales pueden probarse en los sistemas a distancia y digitales e, incluso, reforzarse, dadas las ingentes posibilidades de registrar datos, evidencias e información del proceso de aprendizaje del estudiante y la capacidad de respuesta y realimentación automática o personalizada.

Ante dificultades similares a las vividas durante el confinamiento, que impiden la presencia en un aula de examen, antes de implementar sistemas de proctoring, habría de estudiarse el cómo superar, según país e institución, posibles problemas de carácter técnico, jurídico y ético.

En los estudios superiores de carácter formal y reglado resulta recomendable la aplicación de una evaluación de carácter mixto, a distancia de forma generalizada, síncrona o asíncrona, pero con el agregado de alguna prueba de carácter presencial, hasta tanto se superen las dificultades expuestas. Existen estrategias de evaluación que, sin el uso de esa tecnología de proctoring, muestran una aceptable fiabilidad.

$\mathrm{Y}$, finalmente, tratar de ir modulando las competencias objeto de enseñanza y aprendizaje y, en consecuencia, los procedimientos de evaluación que se irían alejando de pruebas que sólo piden plasmación de lo memorizado que es una forma de invitar a conductas deshonestas. Repensar la evaluación para mejorarla, supondría en su momento, el efecto inmediato de la mejora de nuestras formas de enseñar y de aprender.

\section{REFERENCIAS}

Adachi, C., Tai, J., y Dawson, P. (2018). A Framework for Designing, Implementing, Communicating and Researching Peer Assessment. Higher Education Research and Development, 37(3), 453-467. https://doi.org/10.1080/07294360.2017 .1405913 
Adkins, J., Kenkel, C., y Lim, C. L. (2005). Deterrents to online academic dishonesty. The Journal of Learning in Higher Education, 1(1), 17-22.

Allal, L. (1980). Educational evaluation strategies: Psychopedagogic perspectives and modes of application. Journal for the Study of Education and Development, 3(11), 4-22. https://doi.org/10.1080/021 03702.1980.10821803

Álvarez, I. (2008). Evaluación del aprendizaje en la universidad: una Mirada retrospectiva y prospectiva desde la divulgación científica. Revista Electrónica de Investigación Psicoeducativa, 14(1), 235-272. https://doi.org/10.25115/ejrep. v6i14.1270

Álvarez, V., Padilla, M., Rodríguez, J., Torres, J., y Suárez, M. (2011). Análisis de la participación del alumnado universitario en la evaluación de su aprendizaje. Revista Española de Pedagogía, 69(250), 401-426. https://revistadepedagogia.org

Amo, D., Alier, M., García-Peñalvo, F. J., Fonseca, D., y Casañ, M. J. (2020). Protected Users: A Moodle Plugin to Improve Confidentiality and Privacy Support through User Aliases. Sustainability, 12(6). https://doi. org/10.3390/su12062548

Anderson, T. (2008). Towards a theory of online learning. In T. Anderson y F. Elloumi (Eds.), Theory and practice of online learning (2nd ed., pp. 45-74). AU Press.

Aznarte,J.L., yLacruz,J.M.(2021).Vigilancia automática de exámenes: un gran hermano torpe y peligroso. El País. https://elpais. com/educacion/2021-02-10/vigilanciaautomatica-de-examenes-un-granhermano-torpe-y-peligroso.html

Bangert-Drowns, R. L., Kulik, C.-L.C., Kulik, J. A., y Morgan, M. (1991). The instructional effect of feedback in testlike events. Review of Educational Research, 61(2), 213-238. https://doi. org/10.3102/00346543061002213
Barberà, E. (2006). Aportaciones de la tecnología la e-Evaluación. RED. Revista de Educación a Distancia, 5(6), 1-13. https://revistas.um.es/red/article/ view/270811

Black, P., y Wiliam, D. (1998). Assessment and Classroom Learning. Assessment in Education: Principles, Policy \& Practice, 5(1), 7-74. https://doi. org/10.1080/0969595980050102

Blair, E., y Valdez, K. (2014). Improving higher education practice through student evaluation systems: is the student voice being heard? Assessment \& Evaluation in Higher Education, 39(7), 879-894. https://doi.org/10.1080/02602938.2013 .875984

Blázquez, F., Alonso, L., y Yuste, R. (2017). La evaluación en la era digital. Síntesis.

Brown, S. (2015). La evaluación auténtica: el uso de la evaluación para ayudar a los estudiantes a aprender. RELIEVE, 1(2). https://doi.org/10.7203/ relieve.21.2.7674

Burns, M. (2018). 15 Benefits Of ComputerBased Testing. eLearning Industry. https://elearningindustry.com/15benefits-of-computer-based-testing

Chetwynd, F., y Dobbyn, C. (2011). Assessment, feedback and marking guides in distance education. Open Learning: The Journal of Open, Distance and e-Learning, 26(1), 67-78. https://doi.org $\not 10.1080 / 02680513.2011 .538565$

Chien, S. Y., Hwang, G. J., y Jong, M. S. Y. (2020). Effects of Peer Assessment within the Context of Spherical Video-Based Virtual Reality on EFL Students' EnglishSpeaking Performance and Learning Perceptions. Computers and Education, 146, 1-20. https://doi.org/10.1016/j. compedu.2019.103751

Coll, C., Rochera, M. J., Mayordomo, R. M., y Naranjo, M. (2008). La evaluación continuada como instrumento para el ajuste de la ayuda pedagógica y 
la enseñanza de competencias de autorregulación. Editorial Octaedro.

Dawson, P. (2016). Five ways to hack and cheat with Bring-Your-Own-Device electronic examinations. British Journal of Educational Technology, 47(4), 5926oo. https://doi.org/10.1111/bjet.12246

Day, I. N. Z., van Blankenstein, F. M., Westenberg, P. M., y Admiraal, W. F. (2017). Teacher and student perceptions of intermediate assessment in higher education. Educational Studies. https:// doi.org/10.1080/03055698.2017.138232 4

Deeley, S. (2014). Summative coassessment: A deep learning approach to enhancing employability skills and attributes. Active Learning in Higher Education, 15(1) 39-51. https://doi. org/10.1177/1469787413514649

Dhawan, S. (2020). Online Learning: A Panacea in the Time of COVID-19 Crisis. Journal of Educational Technology Systems, 49(1), 5-22. https://doi. org/10.1177/0047239520934018

Dochy, F., Segers, M., y Sluijsmans, D. (1999). The Use of Self-Peer and Co-Assessment in Higher Education: a Review. Studies in Higher education, 24, 331-350, https:// doi.org/10.1080/0307507991233137993 5

Domenech, J., Blazquez, D., de la Poza, E., y Munoz-Miquel, A. (2015). Exploring the impact of cumulative testing on academic performance of undergraduate students in Spain. Educational Assessment Evaluation and Accountability, 27(2), 153-169. https://doi.org/10.1007/s11092014-9208-Z

Earl, L. M. (2013). Assessment as learning: Using classroom assessment to maximise student learning. Corwin Press.

Espasa, A., Guasch, T., Mayordomo, R. M., Martínez-Melo, M., y Carless, D. (2018). A Dialogic Feedback Index measuring key aspects of feedback processes in online learning environments. Higher Education
Research \& Development, 37(3), 499-513. https://doi.org/10.1080/07294360.2018 .1430125

Falshikov, N. (1986). Product comparisons and process benefits of collaborative peer group and self-assessment. Assessment andHigherEducation,11,146-166, https:// doi.org/10.1080/0260293860110206

Ferguson, P. (2011). Student perceptions of quality feedback in teacher education. Assessment and Evaluation in Higher Education, 36(1), 51-62. https://doi. org/10.1080/02602930903197883

Friedman, A., Blau, I., y Eshet-Alkalai, Y. (2016). Cheating and feeling honest: Committing and punishing analog versus digital academic dishonesty behaviors in higher education. Interdisciplinary Journal of e-Skills and Life Long Learning, 12, 193-205. https://doi. org/10.28945/3629

García Aretio, L. (1987). Rendimiento académico y abandono en la educación superior a distancia. UNED.

García Aretio, L. (1994). Educación a distancia hoy. UNED.

García Aretio, L. (2001). La educación a distancia. De la teoría a la práctica. Ariel.

García Aretio, L. (2017). Algunas adaptaciones requeridas para la evaluación de titulaciones que son impartidas a través de modalidades no presenciales. Informe sobre el estado de la evaluación externa de la calidad en las universidades españolas. ANECA, 101104. https://cutt.ly/flHpQok

García Aretio, L. (2020a). La información sobre los resultados de la evaluación. El feedback. Contextos universitarios mediados. https://aretio.hypotheses. org/4187

García Aretio, L. (2020b). Las tablas de especificación y las rúbricas. Contextos universitarios mediados. https://aretio. hypotheses.org/4115.

García Aretio, L. (2020c). De los exámenes presenciales a los exámenes en línea. 
El fraude. Contextos universitarios mediados. https://aretio.hypotheses. org/4508.

García-Peñalvo, F. J. (2020). Evaluación online: la tormenta perfecta. Ensinar $A$ Distância. https://cutt.ly/wlHpoX7

García-Peñalvo, F. J., Corell, A., AbellaGarcía, V., y Grande, M. (2020). La evaluación online en la educación superior en tiempos de la COVID-19. Education in the Knowledge Society, 21. https://doi. org/10.14201/eks.20202100

Gessa, A. (2011). La coevaluación como metodología complementaria de la evaluación del aprendizaje: análisis y reflexión en las aulas universitarias. Revista de Educación 354, 749-764. http://hdl.handle.net/10272/10762

Gibbs, G., y Simpson, C. (2009). Condiciones para una evaluación continuada favorecedora del aprendizaje. Universitat de Barcelona. Editorial Octaedro. http:// hdl.handle.net/2445/144983

Gikandi, J. W., Morrow, D., y Davis, N. E. (2011). Online formative assessment in higher education: A review of the literature. Computers \& Education, 57, 2333-2351. https://doi.org/10.1016/j. compedu.2011.06.004

Glaser, R. (1963). Instructional technology and the measurement of learning outcomes: Some questions. American Psychologist, 18(8), 519-521. https://doi. org/10.1037/hoo49294

González-González, C. S., Infante-Moro, A., e Infante-Moro, J. (2020). Implementation of E-proctoring in Online Teaching: A Study About Motivational Factors. Sustainability, 12(8). https://doi. org/10.3390/su12083488

Harmon, O. R., y Lambrinos, J. (2008). Are Online Exams an Invitation to Cheat? The Journal of Economic Education, 39(2), 116-125. https://doi.org/10.3200/ JECE.39.2.116-125

Harper, R., Bretag, T., y Rundle, K. (2021). Detecting contract cheating: examining the role of assessment type. Higher Education Research \& Development, 4O(2), 263-278. https://doi.org/10.1080 07294360.2020.1724899

Hills, J. R. (1981). Evaluación y medición en la escuela. Kapeluzs.

Holmes, N.(2017). Engaging with assessment: Increasing student engagement through continuous assessment. Active Learning in Higher Education, 19(1). https://doi. org/10.1177/1469787417723230

Hussey, I. (2017). Continuous assessment in a new Testament survey course: Empirically informed reflections on an Australian trial. Teaching Theology Religion, 2O, 230-242. https://doi. org/10.1111/teth.12391

Kevereski, L. (2017). (Self) evaluation of knowledge in students' population in higher education in Macedonia. Research in Pedagogy, 7(1), 69-75. https://doi. org/10.17810/2015.49

Kornell, N. (2009). Optimising learning using flashcards: Spacing is more effective than cramming. Applied Cognitive Psychology, 23(9). https://doi.org/10.1002/acp.1537

Martin, F., y Ndoye, A. (2016). Using learning analytics to assess student learning in online courses. Journal of University Teaching \& Learning Practice, 13(3), 7. http://ro.uow.edu.au/jutlp/vol13/iss3/7/

McAlpine, M. (2002). Principles of assessment. CAA Centre, University of Luton. https://cutt.ly/OlHd32J

McCarthy, J. (2017). Enhancing feedback in higher education: Students' attitudes towards online and inclass formative assessment feedback models. Active Learning in Higher Education, 18(2), 127-141. https://doi. org/10.1177/1469787417707615

McDonald, B. (2012). Self-assessment and student-centered learning. http://files. eric.ed.gov/fulltext/ED536980.pdf

McLaughlin, T., y Yan, Z. (2017). Diverse delivery methods and strong psychological benefits: A review of online formative 
assessment. Journal of Computer Assisted Learning, 33, 562-574. https:// doi.org/10.1111/jcal.12200

Meyen, E. L., Aust, R. J., Bui, Y. N., e Isaacson, R. (2002). Assessing and monitoring student progress in an e-learning personnel preparation environment. Teacher education and special education, 25(2). 187-198. https:// doi.org/10.1177/088840640202500210

Morgan, C. H., y O’Reilly, M. (1999). Assessing open and distance learners. Kogan y Page.

Nicol, D. (2010). From monologue to dialogue: Improving written feedback processes in mass higher education. Assessment \& Evaluation in Higher Education, 35(5), 501-517. https://doi. org/10.1080/02602931003786559

Nicol, D., y Macfarlane-Dick, D. (2006). Formative assessment and self-regulated learning: A model and seven principles of good feedback practice. Studies in Higher Education, 31(2), 199-218. https://doi. org/10.1080/03075070600572090

Nulty, D. (2011). Peer and self-assessment in the first year of university. Assessment and Evaluation in Higher Education, 36(5), 493-507. https://doi. org/10.1080/02602930903540983

Oren, F. (2018). Self, Peer and Teacher Assessments: What is the Level of Relationship Between them? European Journal of Education Studies, o. https:// doi.org/10.5281/zenodo.1249959

Panadero, E., y Alqassab, M. (2019). An empirical review of anonymity effects in peer assessment, peer feedback, peer review, peer evaluation and peer grading. Assessment \& Evaluation in Higher Education, 44(8), 1253-1278. https://doi. org/10.1080/02602938.2019.1600186

Pérez Juste, R. (1996). La evaluación en la Universidad. En V. García Hoz, La educación personalizada en la Universidad. Rialp, (pp. 431-478).
Pintrich, P. R., y Zusho, A. (2002). Student motivation and self-regulated learning in the college classroom. Higher Education: Handbook of Theory and Research, XVII, 55-128. https://doi.org/10.1007/978-94010-0245-5 2

Popham, W. J. (1983). Evaluación basada en criterios. Magisterio Español.

Prince, D. J., Fulton, R. A., y Garsombke, T. W. (2009). Comparisons of proctored versus non-proctored testing strategies in graduate distance education curriculum. Journal of College Teaching \& Learning, 6(7), 51-63. https://doi.org/10.19030/tlc. v6i7.1125

Quesada, R. (2006). Evaluación del aprendizaje en la educación a distancia "en línea". RED. Revista de Educación a Distancia, 6. https://revistas.um.es/red/ article/view/24291

Robles, M., y Braathen, S. (2002). Online assessment techniques. Delta Pi Epsilon Journal, 44(1). 39-49. https://www. learntechlib.org/p/93435/

Rodríguez, G., e Ibarra, M. S. (Eds.) (2011). $e$-Evaluación orientada al e-Aprendizaje estratégico. Narcea.

Rovai, A. P. (2000). Online and traditional assessments: what's the difference? The Internet and higher education, 3, 141151. https://doi.org/10.1016/S10967516(01)00028-8

Russell, J., Elton, L., Swinglehurst, D., y Greenhalgh, T. (2006). Using the online environment in assessment for learning: a case-study of a webbased course in primary care. Assessment and Evaluation in Higher Education, 31(4), 465-478. https://doi. org/10.1080/02602930600679209

Ryan, S., Scout, B., Freeman, H., y Patel, D. (2000). The Virtual University. Kogan Page.

Salvadores-Merino, C. (2019). Selfassessment vs. external evaluation of proficiency in English as a foreign language among student teachers. 
ReiDoCrea, 8(3), 324-337. https://doi. org/10.30827/Digibug.58464

Sambell, K., Brown, S., y Graham, L. (2018). Professionalism in Practice: Key Directions in Higher Education Learning, Teaching and Assessment. Palgrave Macmillan. https://doi.org/10.1007/9783-319-54552-3

Sanchez, C. E., Atkinson, K. M., Koenka, A. C., Moshontz, H., y Cooper, H. (2017). Self-grading and peer-grading for formative and summative assessments in 3rd through 12th grade classrooms: A meta-analysis. Journal of Educational Psychology, 109(8). https://doi. org/10.1037/eduooo0190

Sangrá, A. (2020). Decálogo para la mejora de la docencia on-line. UOC.

San Martín, S., Jiménez, N., y Jerónimo, E. (2016). La evaluación del alumnado universitario en el Espacio Europeo de Educación Superior. Aula Abierta, 44(1), 7-14. https://doi.org/10.1016/j. aula.2015.03.003

Schrum, L., Burbank, M. D., y Capps, R. (2007). Preparing future teachers for diverse schools in an online learning community: Perceptions and practice. Internet \& Higher Education, 10(3), 204-211. https://doi.org/10.1016/j. iheduc.2007.06.002

Scriven, M. (1967). The methodology of evaluation. In R. W. Tyler, R. M. Gagné, $\mathrm{y}$ M. Scriven (Eds.), Perspectives on curriculum evaluation (Vol. 1, pp. 39-83). Rand McNally.

Shuey, S. (2002). Assessing online learning in higher education. Journal of Instruction Delivery Systems, 16(2), 13-18. https:// cutt.ly/5lHlSNe
Shute, V. J. (2008). Focus on formative feedback. Review of Educational Research, 78(1), 153-189. https://doi. org/10.3102/0034654307313795

Shute, V. J., y Becker, B. J. (2010). Prelude: assessment for the 21 st century. En V. J. Shute y B. J. Becker (Eds.), Innovative Assessment for the 21st Century (pp. 1-11). Springer. https://doi.org/10.1007/978-14419-6530-1 1

Stufflebeanm, D. L., Madaus, G. F., y Kellaghan, T. (2002). Evaluation Models: Viewpoints on Educational and Human Services Evaluation. Kluwer Academic Publishers. https://doi.org/10.1007/o306-47559-6

Thomas, G., Martin, D., y Pleasants, K. (2011). Using self-and peer-assessment to enhance students' future-learning in higher education. Journal of University Teaching and Learning Practice, 8(1), 1-17. https://ro.uow.edu.au/jutlp

Tillema, H. H. (2009). Assessment for learning to teach: appraisal of practice teaching lessons by mentors, supervisors, and students teachers. Journal of Teacher Education, 6o(2), 155-167. https://doi. org/10.1177/0022487108330551

Topping, K. J. (2009). Peer Assessment. TheoryInto Practice, 48(1), 20-27.https:// doi.org/10.1080/00405840802577569

Vallés, C., Ureña, E., y Ruiz, E. (2011). La evaluación formativa en docencia universitaria. Resultados globales de 41 estudios de caso. REDU, 9(1), 135-158. https://doi.org/10.4995/redu.2011.6184

Walvoord, B. E. (2010). Assessment Clear and Simple: A Practical Guide for Institutions, Departments, and General Education. Jossey-Bass. 